Proceeding Series of the Brazilian Society of Computational and Applied Mathematics

\title{
Identificação de Modelo ARX e Controle PI-D de um Conjunto Experimental Correia Rolante e Motor CC
}

\author{
Félix Eduardo Mapurunga de Melo ${ }^{1}$ \\ Clóvis Pontes Façanha ${ }^{2}$ \\ UNIFOR - CCT - Engenharia de Controle e Automação, Fortaleza, CE, \\ José Everardo Bessa Maia ${ }^{3}$ \\ UECE - CCT - Ciência da Computação, Fortaleza, CE
}

\begin{abstract}
Resumo. Este trabalho apresenta o procedimento, os resultados e a validação da identificação de um modelo ARX (autoregressive exogenous) para um conjunto experimental construído em laboratório formado por uma Correia Rolante acionada por Motor CC. Para fins de comparação foi também identificado um modelo de segunda ordem pelo ajuste dos parâmetros da resposta temporal a um degrau de entrada. Para validar o modelo ARX, controladores PI-D discretos para o conjunto foram projetados utilizando os dois modelos e os seus desempenhos foram comparados em ensaios de variação de carga em degrau utilizando o índice NRMSE (normalized root mean square error). Os resultados mostram que o controlador PI-D projetado com base no modelo ARX apresentou desempenho superior indicando sua melhor adequação para este projeto.
\end{abstract}

Palavras-chave. Identificação de Sistemas, Modelo ARX, Sistemas Dinâmicos, Controle PI-D, Controle de Motor CC.

\section{Introdução}

Identificação de sistemas consiste na modelagem matemática de um sistema dinâmico a partir do comportamento entrada-saída do sistema físico. Este procedimento exige a definição de uma classe de modelos e um critério de seleção de modelos adequado para a classe escolhida. O objetivo final é aproximar o sistema físico cujo modelo exato é desconhecido por uma equação a diferenças (ou diferencial) utilizando os dados experimentais disponíveis $[4,7]$.

Identificação de sistemas adquire maior importância devido a crescente complexidade dos processos de produção e a demanda por alta eficiência em processos industriais, o que força os sistemas de controle associados a possuírem maior robustez e precisão. Visando

\footnotetext{
${ }^{1}$ edumapurunga@gmail.com

2 clovis.facanha@hotmail.com

3 jose.maia@uece.br
} 
o aprimoramento dos sistemas de controle para responder a demanda destas aplicações, faz-se necessário extrair maior conhecimento sobre as plantas a serem controladas [1].

Por outro lado, os processos industriais com os quais a engenharia trabalha muitas vezes são complexos em demasia para a obtenção satisfatória de um modelo determinístico a partir das leis físicas, fazendo-se necessárias várias aproximações e linearizações. A identificação de sistemas torna-se assim um elo entre as aplicações do mundo real e o mundo matemático da teoria de controle e suas abstrações [4].

Neste estudo, um modelo ARX (autoregressive exogenous) é identificado para um conjunto experimental constituído de Correia Rolante acionada por um Motor de Corrente Contínua (CC). Para validar o modelo ARX, também foram identificados modelos de primeira e de segunda ordens pelo ajuste dos parâmetros da resposta temporal a um degrau de entrada. Então, controladores PI-D (Proporcional+Integral-Derivativo) discretos para o conjunto foram projetados utilizando os dois modelos e os seus desempenhos foram comparados utilizando o índice NRMSE (normalized root mean square error) na resposta a variações de carga em degrau.

Na Seção 2 é apresentada uma breve revisão de identificação de modelos ARX, identificação de modelos de primeira e segunda ordens pela resposta temporal e controle PI-D. Na Seção 3 são apresentados e discutidos os resultados e o artigo é concluído na Seção 4.

\section{Identificação e Controle de Sistemas}

\subsection{Modelos ARX e Estimação por Mínimos Quadrados Médio}

A determinação do modelo é feita através da descrição do sistema como uma equação a diferenças, que relaciona a entrada $u(k)$ com saída $y(k)$ da planta. Um modelo genérico de sistema pode ser representado pela equação $A(q) y(k)=B(q) u(k)+v(k)$, na qual $q^{-1}$ é o operador de atraso unitário, de forma que $y(k) * q^{-n}=y(k-n), v(k)$ é um ruído branco com média zero e variância $\sigma^{2}$, não correlacionado com a entrada [4]. Os polinômios $A(q)$ e $B(q)$ são da forma $A(q)=1+a_{1} q^{-1}+a_{2} q^{-2}+\ldots+a_{n_{a}} q^{-n_{a}}$ e $B(q)=b_{1} q^{-1}+b_{2} q^{-2}+\ldots+b_{n_{b}} q^{-n_{b}}$.

O modelo em questão é conhecido como Autoregressivo com entrada exógena (ARX Autoregressive with exogenous input) $[1,4,7]$. Em notação matricial, mais compacta:

$$
y(k)=\varphi^{T}(k) \theta+v(k),
$$

em que $\theta^{T}=\left[\begin{array}{llllllll}a_{1} & a_{2} & a_{3} & \ldots & a_{n_{a}} & b_{1} & \ldots & b_{n_{b}}\end{array}\right]$ e $\varphi^{T}=\left[\begin{array}{llll}-y(k-1) & -y(k-2) & \ldots & -y(k-\end{array}\right.$ $\left.\left.n_{a}\right) u(k-1) \ldots u\left(k-n_{b}\right)\right]$, com $\theta$ sendo o vetor de parâmetros a estimar, enquanto que o vetor $\varphi$ contém os dados do sistema. Assim, um conjunto de dados de tamanho $N$ pode ser representado, em notação matricial, por:

$$
Y_{N}^{T}=[y(1) y(2) \ldots y(N)], \quad \phi_{N}^{T}=[\varphi(1) \varphi(2) \ldots \varphi(N)] .
$$

O vetor de parâmetros pode ser obtido através da estimação por mínimos quadrados médio pela seguinte equação [1]:

$$
\widehat{\theta}_{N}=\left[\phi_{N}^{T} \phi_{N}\right]^{-1} \phi_{N}^{T} Y_{N}
$$




\subsection{Identificação paramétrica pela resposta temporal ao degrau}

Utilizando métodos clássicos, foram identificados modelos de primeira e de segunda ordens para contrastar com o modelo ARX quando utilizados no controle PI-D. O primeiro foi um modelo de primeira ordem com atraso de transporte (fodt - First Order Delay Time). Este modelo pode ser uma boa aproximação para representar sistemas de ordem superior por um sistema de primeira ordem com tempo morto $[6,7]$. A função de transferência é dada por:

$$
G_{p}(s)=\frac{K}{(\tau s+1)} e^{-t_{d} s},
$$

onde $K$ é o ganho estático, $t_{d}$ é o atraso de transporte e $\tau$ é uma constante de tempo. $\mathrm{O}$ ganho estático é de fácil determinação. Vários métodos já foram propostos para determinar o atraso de transporte $t_{d}$ e a constante de tempo $\tau$. O primeiro método baseado em pontos sobre a curva de reação foi proposto por Smith (1972), citado em [7], e foi aqui adotado. O método seleciona os tempos em que a resposta alcança $t_{1}=28,3 \%$ e $t_{2}=63,2 \%$ do valor final estabilizado da resposta ao degrau de malha aberta e resolve o sistema de equações $\tau=a t_{1}+b t_{2}, t_{m}=c t_{1}+d t_{2}, \operatorname{com} a=-1,5, b=1,5, c=1,5$ e $d=-0,5$. O atraso de transporte $t_{d}$ pode ser determinado visualmente pelo atraso entre o momento de aplicação do degrau de entrada e o início da resposta.

O modelo de segunda ordem parte da função de transferência canônica de segunda ordem

$$
G_{p}(s)=\frac{K \omega_{0}^{2}}{s^{2}+2 \zeta \omega_{0} s+\omega_{0}^{2}},
$$

onde $K$ é o ganho, $\omega_{0}$ é a frequência natural não amortecida e $\zeta$ é o coeficiente de amortecimento. A determinação de $K, \omega$ e $\zeta$ pode ser feita a partir dos pontos de máximos e mínimos locais da resposta ao degrau utilizando o seguinte conjunto de equações [7]:

$$
\left\{\begin{array}{l}
t_{k}=k \frac{\pi}{\omega_{0} \sqrt{1-\zeta^{2}}}, \quad k=1,2,3, \ldots \\
y\left(t_{k}\right)=K\left[1-(-1)^{k} M^{k}\right] \\
M=\exp \left[-\zeta \pi \sqrt{1-\zeta^{2}}\right]
\end{array}\right.
$$

\subsection{Controle PI-D discreto}

Controladores PID são frequentemente utilizados na indústria devido ao seu desempenho satisfatório associado a facilidade de sintonizar derivada do pequeno número de parâmetros ajustáveis [1]. A ação de controle do controlador PID discreto, em função do erro, na forma de velocidade, é dada pela equação $[2,8]$

$$
M(z)=-K_{P} E(z)+K_{I} \frac{E(z)}{1-z^{-1}}-K_{D}\left(1-z^{-1}\right) E(z),
$$

na qual $E(z)$ é como definido na Seção 3.3 .

Entretanto, note que a ação derivativa atuando sobre o erro pode gerar pulsos de grande amplitude, uma situação indesejável em qualquer aplicação prática. O controlador PI-D é um controlador PID modificado no qual a ação derivativa atua apenas sobre a 
realimentação da saída conforme o diagrama da Figura 3(a). Nesta configuração as ações de controle são dadas pelo conjunto de equações [8]

$$
\left\{\begin{aligned}
u_{P I}(z) & =\left(K_{P}-\frac{K_{I} T}{2}+\frac{K_{I} T}{1-z^{-1}}\right) E(z), \\
u_{D}(z) & =K_{D} \frac{2}{T} \frac{1-z^{-1}}{1+z^{-1}} Y(z) .
\end{aligned}\right.
$$

Em função desta propriedade, este estudo adotou o controlador PI-D. É fácil mostrar que essa modificação não altera a função de transferência entre a entrada de distúrbio e a saída.

\section{Resultados e discussão}

\subsection{Conjunto experimental Correia Rolante-Motor CC}

O conjunto é constituído de motor CC de campo constante com redutor, microcontrolador Arduino, driver de potência e correia rolante acoplada conforme a Figura 1(a). O motor utilizado tem alta rotação nominal de 10.000 rpm e está acoplado com caixa redutora de 43:1. Fez parte do projeto construir e testar o protótipo eletromecânico da correia rolante incluindo o acoplamento do motor de corrente contínua com codificador para aquisição de dados de velocidade. Foram coletados 500 pontos das respostas ao degrau e a uma entrada PRBS (pseudo-random binary sequence) com período de amostragem de 4,97 ms. Foram feitas quatro coletas independentes para assegurar consistência dos dados. A Figura 1(b) mostra uma fotografia da bancada de teste.

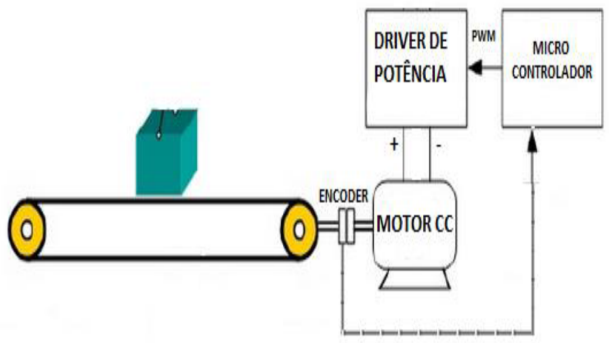

(a) Diagrama de blocos do conjunto experimental.

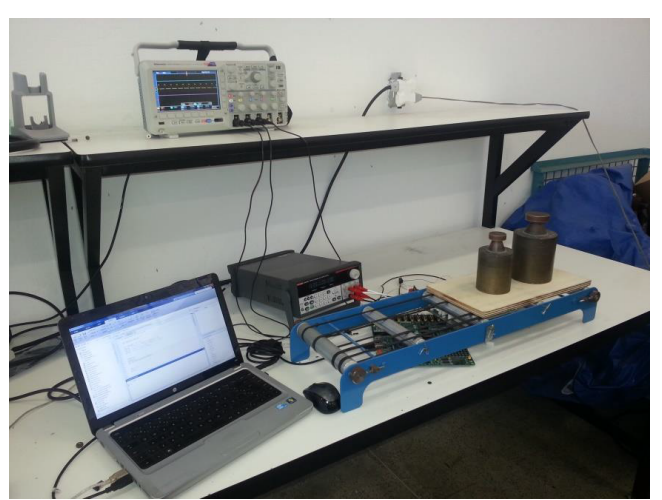

(b) Bancada experimental.

Figura 1: Conjunto experimental.

\subsection{Modelos identificados}

A ferramenta Sisotool do Control System Toolbox [3] foi utilizada no projeto ou na validação dos modelos identificados nesta seção. Utilizando os dados experimentais obtidos 
como descrito na Subseção 3.1 os modelos da função de transferência de malha aberta identificados, de primeira e segunda ordens, foram, respectivamente: $G_{m a}(s)=\frac{21,6}{s+25,86} e^{-9,94 s}$ e $G_{m a}(s)=\frac{20,42 s+2395}{s^{2}+100,8 s+2867}$. O valor $t_{d}=9,94$ foi determinado visualmente como sendo igual dois períodos de amostragem. A implementação discreta não está mostrada por falta de espaço. Por sua vez, a equação recursiva do Modelo ARX resultou em:

$$
y(k)=0,433 y(k-1)+0,433 y(k-2)+0,2438 u(k-1),
$$

que resulta na função de transferência discreta,

$$
G_{P}(z)=\frac{0,24 z^{-1}}{1-0,43 z^{-1}-0,43 z^{-2}} .
$$

A verificação das respostas do sistema real em malha aberta na Figura 2a e da aproximação entre as respostas dos modelos estimados e do sistema real na Figura 2b são um passo intermediário importante pois comprovam o domínio do procedimento experimental.

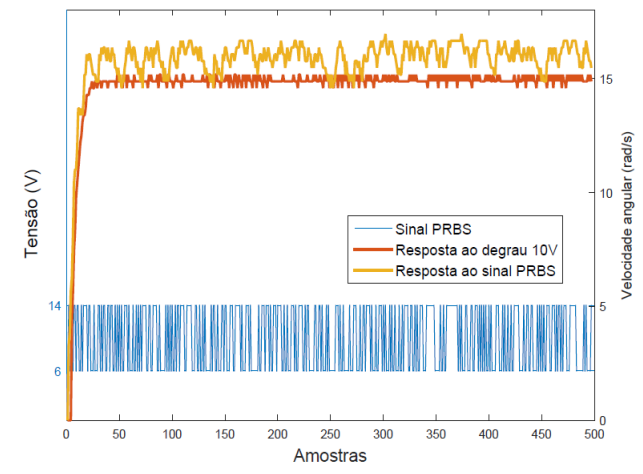

(a) Respostas ao degrau e PRBS do conjunto.

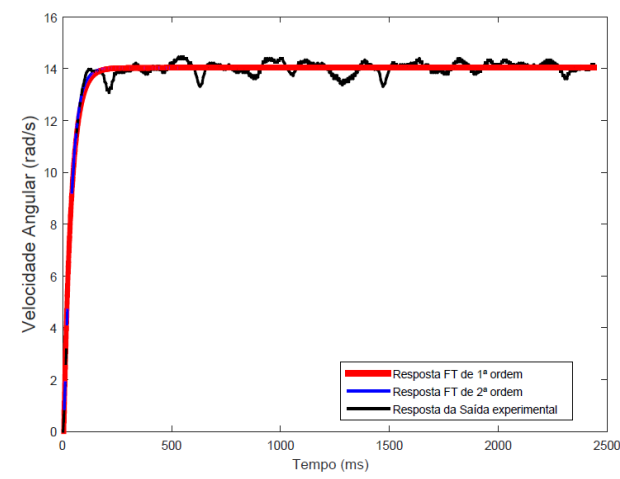

(b) Respostas das funções estimadas.

Figura 2: Respostas ao degrau em malha aberta do sistema e das funções estimadas.

\subsection{Controle PI-D do Conjunto Correia Rolante - Motor CC}

A topologia do controle PI-D é a mostrada na Figura 3a [5, 8]. O projeto do controle PI-D resultou nas seguintes funções de transferência discretas para os blocos PI e D.

A equação a diferenças do controle PI resultou em

$$
u_{P I}(k)=u_{P I}(k-1)+0,547 e(k)-0,4243 e(k-1),
$$

que resulta na função de transferência discreta do Controle PI:

$$
G_{P I}(z)=\frac{0,5471 z-0,4243}{z-1} .
$$

A Função de transferência do Controle D foi:

$$
G_{D}(z)=\frac{0,39(z-1)}{(z+1)} .
$$




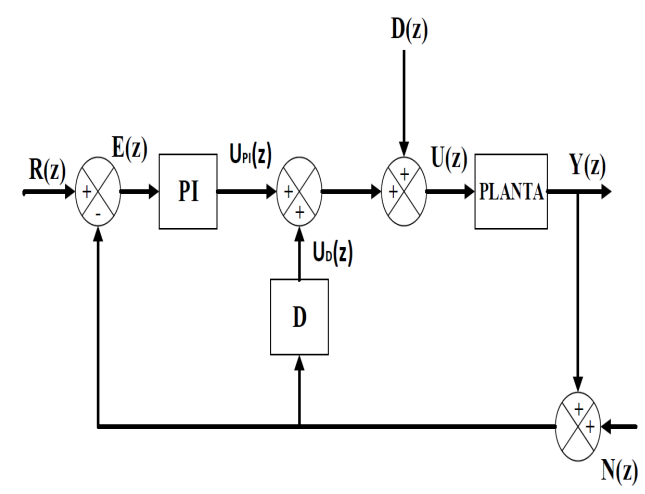

(a) Topologia do controlador PI-D $[5,8]$.

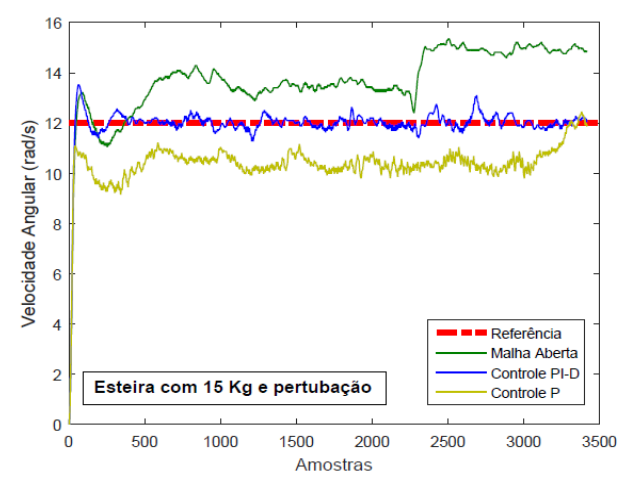

(b) Respostas a um degrau de carga.

Figura 3: Modelo do controlador e o efeito sobre a resposta a um degrau de carga.

\subsection{Discussão dos resultados}

Os resultados na Tabela 1 mostram o desempenho superior do projeto utilizando o modelo ARX. Também, o conjunto experimental de baixo custo construído no laboratório mostrou-se um sistemas fortemente ruidoso como pode-se ver da resposta ao degrau em vazio na Figura 3(b). Esta figura mostra que as imperfeições na construção da correia rolante dá origem a um ruído de carga permanente. Isso se deve a que níveis diferentes de atrito e de pressão das correias sobre os cilindros, além das imperfeições no alinhamento dos cilindros variam a carga durante o ciclo de giro da correia.

Na Figura 3(b) a correia rolante parte com uma carga de $15 \mathrm{Kg}$. Um degrau de carga é aplicado após 2,30 segundos quando o peso cai para fora da correia (sai da correia). A Figura 3(b) mostra as respostas para malha aberta, controle proporcional e controle PI-D utilizando o modelo ARX identificado. Esta figura mostra que a combinação controle PI-D com o modelo ARX atuou eficazmente na compensação do degrau de carga.

Tabela 1: Desempenho do controle PI-D utilizando os diversos modelos.

\begin{tabular}{|r|l|r|}
\hline Modelo & MSE & NRMSE \\
\hline ARX $(2,1,1)$ & 0,50 & 0,06 \\
Ordem 1 & 1,70 & 0,43 \\
Ordem 2 & 1,10 & 0,26 \\
\hline
\end{tabular}

\section{Conclusão}

Foram apresentados os resultados de um projeto de construção experimental em laboratório de um conjunto Correia Rolante - Motor CC de campo constante e a identificação e controle PI-D do conjunto. Foram identificados três modelos: um modelo de função de transferência de primeira ordem com atraso de transporte, um modelo de função de 
transferência de segunda ordem e um modelo ARX de segunda ordem idenficado pelo método dos mínimos quadrados médio. Na aplicação dos modelos de segunda ordem para o controle PI-D eles foram utilizados em cascata com o atraso de transporte identificado para o modelo de primeira ordem. Note que o procedimento experimental impõe que o atraso de transporte seja identificado como um múltiplo do período de amostragem, embora possivelmente não corresponda ao atraso físico real. Porém, isto não afeta o modelo já que a implementação é discreta utilizando o mesmo período de amostragem.

Em conclusão, o controle projetado utilizando o modelo $\operatorname{ARX}(2,1,1)$ mostrou-se superior aos outros dois, projetados utilizando os modelos de primeira e segunda ordens obtidos por identificação paramétrica, reduzindo para 30\% e 45\%, respectivamente, o NRMSE na resposta a um distúrbio de carga em degrau.

\section{Referências}

[1] L. A. Aguirre, Introdução à Identificação de Sistemas, $3^{a}$ Ed., Ed. UFMG, (2007).

[2] J. Kon, Y. Yamashita, T. Tanaka, A. Tashiro and M. Daiguji, Practical application of model identification based on ARX models with transfer functions. Control Engineering Practice, 21(2), 195-203, (2013).

[3] L. Ljung, System identification toolbox: User's guide. MathWorks Incorporated, (1995).

[4] L. Ljung, System identification: theory for the user, PTR Prentice Hall, Information and System Sciences Series 198 (1987).

[5] K. Ogata, Discrete-time control systems, 2nd Ed., Englewood Cliffs, NJ: Prentice Hall, (1995).

[6] J. Schoukens, R. Pintelon and Y. Rolain, Mastering System Identification in 100 Exercises, IEEE Press and Jonh Wiley and Sons, INC., (2012).

[7] T. Söderström and P. Stoica, System Identification, Prentice-Hall International, Hemel Hempstead, UK, (1989).

[8] K. S. Tang, K. F. Man, G. Chen and S. Kwong, An optimal fuzzy PID controller. Industrial Electronics, IEEE Transactions on, 48(4), 757-765, (2001). 